\title{
OPTIMAL AND QUASIOPTIMAL ALGORITHMS OF DISTINCTION OF THE COMPRESSED IMAGES IN BASES OF ORTHOGONAL POLYNOMIALS
}

The synthesis and analysis of effective practically realized algorithms of distinction of signals and images represented in bases of orthogonal polynomials was executed. Influence of distortions at formation of sensed and reference useful signals was considered. Recommendations about application of the synthesized distinction algorithms were formulated.

Keywords: Signal and image distinction, generalized signal spectrum, maximum - likelihood receiver, incomplete signal reception probability of signal distinction.

\section{Introduction}

Modern processing systems of the graphic and multimedia information (supervision of various objects, remote monitoring, control and aiming, etc.) should solve a problem of the operative analysis of complex dynamic images and transmission of information flows of a various type. In all these information structures the procedures of signal compression, signal restoration, parameters' measurement, recognition, etc are present. In a number of papers $[1-3]$ it is shown that for the decision of many problems mentioned above the application of transformation of the initial image on bases of orthogonal polynomials or the functions connected with them is effective. In the present work the possibility of distinction of the images transformed thereby with use of the theory of statistical decisions is considered.

\section{Problem statement}

Let's assume that the realization of the two-dimensional random field $\xi(r), r=(x, y)$, which can be or an additive mix of the useful signal $s_{1}(r)$ and hindrance $\eta(r): \xi(r)=s_{1}(r)+\eta(r)$ (hypothesis $\left.H_{1}\right)$ or an additive mix of the useful signal $s_{2}(r)$ and hindrance $\eta(r): \xi(r)=s_{1}(r)+\eta(r)$ (hypothesis $H_{2}$ ), incomes to the receiver input. Herewith the hypothesis $H_{1}$ is realized with probability $p_{1}$, and the hypothesis $\mathrm{H}_{2}$ is realized with probability $p_{2}$.

Let's consider that initial useful signals $s_{1}(r), s_{2}(r)$ can be expand into series of kind

$$
\begin{aligned}
& s_{1}(r)=\sum_{m=0}^{\infty} \sum_{n=0}^{\infty} A_{m n} \varphi_{m n}(r), \\
& s_{2}(r)=\sum_{m=0}^{\infty} \sum_{n=0}^{\infty} B_{m n} \varphi_{m n}(r)
\end{aligned}
$$

in one or another system of orthogonal functions $\left\{\varphi_{m n}(r)\right\}$. Here

$$
\begin{aligned}
& A_{m n}=\int_{\Omega} s_{1}(r) \varphi_{m n}(r) d r, \\
& B_{m n}=\int_{\Omega} s_{2}(r) \varphi_{m n}(r) d r
\end{aligned}
$$

are expansion factors, and $\Omega$ is area of orthogonality of basis $\left\{\varphi_{m n}(r)\right\}$.

It should be noted that useful signals in the process of transformation, compression and the subsequent restoration can be exposed to various restrictions. Firstly, the transmitting device can transmit signals with the limited number of modes $M(M<\infty)$ to the communication channel:

$$
\begin{aligned}
& s_{1}(r) \approx s_{1 M}(r)=\sum_{m, n=0}^{M} A_{m n} \varphi_{m n}(r), \\
& s_{2}(r) \approx s_{2 M}(r)=\sum_{m, n=0}^{M} B_{m n} \varphi_{m n}(r)
\end{aligned}
$$

Secondly, depending on capacity of computing systems as a part of the receiver-analyzer, computational burden (computer timetable) etc not all modes of signals (1) but only the most powerful ones can be processed. In both cases so-called incomplete processing of a useful signal takes place.

Models of hindrances at optical and optoelectronic processing of the information are in detail considered in [4, 5]. Generally the additive hindrance hits in information system both at a stage of transformation of analogue signals $s_{1}(r), s_{2}(r)$ in the discrete form and from the communication channel to the receiver-analyzer input. If a quantum character of weak optical signals is not taken into consideration and signals is assumed as intensive enough then it

\footnotetext{
* Oleg Vyacheslavovich Chernoyarov ${ }^{1}$, Martin Breznan ${ }^{2}$

${ }^{1}$ Department of Radio Engineering, National Research University, Moscow Power Engineering Institute, Russia, E-mail: o_v_ch@mail.ru

${ }^{2}$ Faculty of Electrical Engineering, Department of Telecommunications and Multimedia, University of Zilina, Slovakia
} 
is possible often to believe, that a hindrance is Gaussian random field with known mathematical expectation $M \eta=\langle\eta(r)\rangle$ and cor-

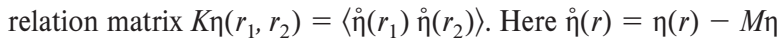
is a centered random field. As the mathematical expectation $M \eta$ is a priori known than it can be subtracted from a hindrance $\eta(r)$ in the processing operations and it can not be taken into consideration at the analysis. Thereupon a symbol « $०$ » over $\eta(r)$ for simplification of record will be skipped further.

Spectral-correlation properties of a field $\eta(r)$ can be various depending on physical statement of a problem $[4,5]$. If processing algorithms don't consider thin difference of spatial spectrums of a useful signal and a hindrance but focus the attention on the analysis of generalized spectrum's modes of the field $s_{i}(r), i=1,2$ than hindrance $\eta(r)$ may be approximated by Gaussian white noise with a correlation matrix of a kind $K \eta\left(r_{1}, r_{2}\right)=\left(N_{0} / 2\right) \delta\left(r_{2}-r_{1}\right)$. The model of white noise is quite proved if hindrance sources are wideband processes in electronic devices [6]

\section{Optimal and Quasioptimal Algorithms of Images' Distinction in Bases of Orthogonal Polynomials}

According to [7] the algorithm of distinction of signals $s_{i}(r)$, $=1,2$ on the background of noise $\eta(r)$ can be presented in the general form as follows:

$$
L_{<}^{>} h_{0},
$$

where $L$ is a logarithm of functional of likelihood ratio (FLR) for the hypothesis $H_{1}$ against alternative $H_{2}$, and $h_{0}$ is the threshold calculated according to chosen optimality criterion. If logarithm of FLR exceeds the threshold $h_{0}$ then the decision on signal presence $s_{1}(r)$ in realization of the observable data $\xi(r)$ is delivered and otherwise - on signal presence $s_{2}(r)$.

Believing a hindrance $\eta(r)$ by Gaussian white noise for logarithm of FLR we have [7]

$$
L=\frac{2}{N_{0}} \int_{\Omega} \xi(r)\left[s_{1}(r)-s_{2}(r)\right] d r-\frac{E_{1}-E_{2}}{N_{0}} .
$$

Here $E_{i}=\int_{\Omega} s_{i}^{2}(r) d r, i=1,2$ is a total energy of signal $s_{i}(r)$. Then the decision rule (4) will be presented in the form

$$
\frac{2}{N_{0}} \int_{\Omega} \xi(r)\left[s_{1}(r)-s_{2}(r)\right] d r-\frac{E_{1}-E_{2}}{N_{0}}>h_{0} .
$$

At incomplete reception when the mix $\xi(r)=s_{i M}(r)+\eta(r)$ with unknown number of modes $M$ at a useful signal (3) is observed for the hypothesis $H_{i}$ we receive from Eq. (6)

$$
L=\frac{2}{N_{0}} \int_{\Omega} \xi(r)\left[s_{1}(r)-s_{2}(r)\right] d r-\frac{E_{1}-E_{2}}{N_{0}}>h_{0} .
$$

According to [7] the detection algorithm (7) will not be optimal now. We name Eq. (7) as quasioptimal decision rule. Really, Eq. (7) turned into optimal decision rule (6) at $M \rightarrow \infty$. Comparing characteristics of algorithms (6) and (7) it is possible to estimate losses in efficiency of processing algorithm (7) and to define requirements to throughput of a communication channel.

If $v$ modes for representation of reference signals $s_{1}(r), s_{2}(r)$ on the receiving site is used then the decision rule (6) will be transformed to form

$$
L=\frac{2}{N_{0}} \int_{\Omega} \xi(r)\left[s_{1 v}(r)-s_{2 v}(r)\right] d r-\frac{E_{1 v}-E_{2 v}>}{N_{0}}<h_{0}
$$

where $E_{i v}=\int_{\Omega} s_{i v}^{2}(r) d r, i=1$, 2. Signals $s_{i v}(r)$ in Eq. (8) containing $v$ modes are defined similarly Eq. (3).

Generally the number $v$ can be both more and less $M$. It is determined by restriction on computing possibilities of the receiveranalyzer, the prior information on channel throughput and other restrictions on structure of information transmission and processing system.

In a number of practical applications there can be a notation of Eqs. (4), (5) in a vector-matrix form more convenient. We will designate

$$
\begin{aligned}
X_{m n} & =\int_{\Omega} \xi(r) \varphi_{m n}(r) d r, \\
C_{m n}^{-} & =A_{m n}-B_{m n}, C_{m n}^{+}=A_{m n}+B_{m n} .
\end{aligned}
$$

Then it is possible to write down Eqs. (4), (5) as

$$
L=\frac{1}{N_{0}} \sum_{m, n=0}^{\infty}\left(2 X_{m n}-C_{m n}^{+}\right) C_{m n}^{-}>h_{0} .
$$

The similar notation can be offered for algorithms (7), (8).

\section{Efficiency of Image Distinction Algorithms in Bases of Orthogonal Polynomials}

As the quantitative characteristic of image distinction algorithms synthesized in item 3 the mean error probability will be used [7]. We will find the error probability of signal distinction at optimal reception according to algorithm (6) at first. Following [7] the mean error probability of distinction $P_{e}$ we will write down in a form

$$
P_{e}=p_{1} P(2 \mid 1)+p_{2} P(1 \mid 2),
$$

where $p_{i}$ is the prior probability of the hypothesis $H_{i}$ and $P(i \mid j)$ is the probability of decision in favour of $i$-th signal while $j$-th hypothesis was true $(i, j=1,2)$. By definition [7]

$$
P(2 \mid 1)=\int_{-\infty}^{h_{0}} w_{1}(x) d x, P(1 \mid 2)=\int_{h_{0}}^{\infty} w_{2}(x) d x .
$$

Here $w_{i}(x), i=1,2$ is the probability density of logarithm of FLR (5) at hypothesis $H_{i}$.

According to [7] logarithm of FLR L (5) is a Gaussian random value. Then for probabilities (10) is: 


$$
\begin{aligned}
& P(1 \mid 2)=1-\Phi\left[\left(h_{0}-m_{2 L}\right) / \sigma_{2 L}\right], \\
& P(2 \mid 1)=\Phi\left[\left(h_{0}-m_{1 L}\right) / \sigma_{1 L}\right],
\end{aligned}
$$

Through $m_{1 L}, m_{2 L}$ and $\sigma_{1 L}, \sigma_{2 L}$ in Eq. (11) mathematical expectations and mean square deviations of random value $\mathrm{L}(5)$ at hypotheses $H_{1}$ and $H_{2}$ are designated. Implementing immediate averaging of Eq. (5) on all possible realizations of the observable data $\xi(r)$ we can find following expressions for $m_{1 L}, m_{2 L}, \sigma_{1 L}, \sigma_{2 L}$ :

$$
\begin{aligned}
& m_{1 L}=-m_{2 L}=\left(E_{1}+E_{2}-2 R \sqrt{E_{1} E_{2}}\right) / N_{0}, \\
& \sigma_{1 L}^{2}=-\sigma_{2 L}^{2}=2\left(E_{1}+E_{2}-2 R \sqrt{E_{1} E_{2}}\right) / N_{0} .
\end{aligned}
$$

Here is the correlation factor reflecting similarity of the geometrical form of two objects.

Let's introduce designations $m=\left(E_{1}+E_{2}-2 R \sqrt{E_{1} E_{2}}\right) / N_{0}$, $D=2\left(E_{1}+E_{2}-2 R \sqrt{E_{1} E_{2}}\right) / N_{0}$. Then, substituting (12) in (11), and (11) in (9), for the mean error probability of distinction we can write down

$$
P_{e}=p_{1}\left[1-\Phi\left(\frac{h_{0}+m}{\sqrt{D}}\right)\right]+p_{2} \Phi\left(\frac{h_{0}+m}{\sqrt{D}}\right)
$$

The formula (13) becomes simpler if signals $s_{1}(r)$ and $s_{2}(r)$ are equiprobable (prior probabilities of hypotheses $H_{1}$ and $H_{2}$ are equal): $p_{1}=p_{2}=0,5$ and the threshold according to criterion of the ideal observer [7] is chosen $\left(h_{0}=0\right)$ :

$$
P_{e}=1-\Phi\left(\frac{m}{\sqrt{D}}\right)=1-\Phi\left(\frac{\sqrt{z_{1}^{2}+z_{2}^{2}-2 z_{1} z_{2} R}}{2}\right)
$$

Here $z_{1,2}^{2}=2 E_{1,2} / N_{0}$. If in addition to the discriminating signals are symmetric, i.e. $E_{1}=E_{2}=E\left(z_{1}=z_{2}=z\right)$ than

$$
P_{e}=1-\Phi[z \sqrt{(1-R) / 2}] .
$$

Let's come to a case of distinction of two signals (3) limited on number of modes now. But formed reference signal assumes processing of infinite number of modes of the observable field $\xi(r)$. If representation (3) is performed then logarithm of FLR (7) will be Gaussian random value with parameters

$$
m_{1 L}=\frac{2}{N_{0}}\left(\sum_{m, n=0}^{M} A_{m n}^{2}-\sum_{m, n=0}^{M} A_{m n} B_{m n}\right)-\left(E_{1}-E_{2}\right) / N_{0}
$$

$\sigma_{1, L}^{2}(12)$ at the hypothesis $H_{1}$ and

$$
m_{2 L}=\frac{2}{N_{0}}\left(\sum_{m, n=0}^{M} A_{m n} B_{m n}-\sum_{m, n=0}^{M} B_{m n}^{2}\right)-\left(E_{1}-E_{2}\right) / N_{0}
$$

$\sigma_{2, L}^{2}(12)$ at the hypothesis $H_{2}$.

We designate

$$
\begin{aligned}
& \varepsilon_{1 M}=\sum_{m, n=0}^{M} A_{m n}^{2} / \sum_{m, n=0}^{\infty} A_{m n}^{2}, \\
& \varepsilon_{2 M}=\sum_{m, n=0}^{M} B_{m n}^{2} / \sum_{m, n=0}^{\infty} B_{m n}^{2},
\end{aligned}
$$

$$
R_{M}=\varepsilon_{1 M}=\sum_{m, n=0}^{M} A_{m n} B_{m n} / \sqrt{\sum_{m, n=0}^{\infty} A_{m n}^{2} \sum_{m, n=0}^{\infty} B_{m n}^{2}},
$$

take into account that $E_{1}=\sum_{m, n=0}^{\infty} A_{m n}^{2}, E_{2}=\sum_{m, n=0}^{\infty} B_{m n}^{2}$ and overwrite Eqs. (16), (17) as

$$
\begin{aligned}
& m_{1 L}=\left[E_{1}+E_{2}-2 R_{M} \sqrt{E_{1} E_{2}}-2 E_{1}\left(1-\varepsilon_{1 M}\right)\right] / N_{0} \\
& m_{2 L}=-\left[E_{1}+E_{2}-2 R_{M} \sqrt{E_{1} E_{2}}-2 E_{2}\left(1-\varepsilon_{2 M}\right)\right] / N_{0}
\end{aligned}
$$

Then according to Eq. (9) the mean error probability $P_{e}$ of distinction of two signals (3) will be defined as follows

$$
P_{e}=p_{1}\left[1-\Phi\left(\frac{h_{0}-m_{2 L}}{\sigma_{2 L}}\right)\right]+p_{2} \Phi\left(\frac{h_{0}-m_{1 L}}{\sigma_{1 L}}\right) .
$$

The formula (19) becomes simplest if $h_{0}=0, p_{1}=p_{2}=0,5$, $E_{1}=E_{2}=E, \varepsilon_{1 M}=\varepsilon_{2 M}=\varepsilon_{M}$. In this case

$$
P_{e}=1-\Phi\left[z\left(\varepsilon_{M}-R_{M} / \sqrt{2(1-R)}\right],\right.
$$

where $z^{2}=2 E / N_{0}$. At $M \rightarrow \infty$ we have: $\varepsilon_{M} \rightarrow 1, R_{M} \rightarrow R$ and expression (20) proceed to (15). It may be noted that performance degradation of distinction (20) in comparison with a case of optimal reception (15) takes place if

$$
\begin{aligned}
& \sqrt{1-R}>\left(\varepsilon_{M}-R_{M}\right) / \sqrt{1-R} \text { or } \\
& 1-R-\left(\varepsilon_{M}-R_{M}\right)>0 .
\end{aligned}
$$

From a Cauchy-Bunyakowsky-Schwartz inequality [8] follows that

$$
\sum_{m, n=0}^{M} A_{m n} B_{m n} \leq \sqrt{\left(\sum_{m, n=0}^{M} A_{m n}^{2}\right)\left(\sum_{m, n=0}^{M} B_{m n}^{2}\right)} \text { or } R_{M} \leq \varepsilon_{M} .
$$

Consequently the inequality (21) may be broken for strongly correlated images, when $R \rightarrow 1$, and distinction with use of limited number of modes becomes more preferable than with use of unlimited number of modes.

Consider an illustration of the offered approach to distinction of signals/images by giving an example. For simplification of mathematical calculations and visualization of received results we will believe that discriminated signals are one-dimensional. For twodimensional signals the general conclusions will be similar.

For definiteness we will assume that

$$
s_{1}(x)=\exp \left(-\alpha^{2} x^{2}\right),
$$

$s_{2}(x)=s_{1}(x-\tau)$ and correlation factor $\mathrm{R}(12)$ is $R=R(\tau)=$ $=\exp \left(-\alpha^{2} \tau^{2} / 2\right)$. Here $\alpha$ is the parameter characterizing the duration of discriminated signals. As system of orthogonal functions [10] we will choose Hermitian functions [9]: 


$$
\varphi_{n}(t)=\frac{1}{\sqrt{2^{n} n ! \sqrt{\pi}}} H_{n}(t) \exp \left(-\frac{t^{2}}{2}\right) .
$$

Here $H_{n}(t)$ is a Hermitian polynomial of n-th order [9]. Then for the generalized spectrums $A_{n}, B_{n}(2)$ of signals $s_{1}(x), s_{2}(x)$ it can be found:

$$
\begin{aligned}
& A_{n}=\frac{(-1) \sqrt[n / 2]{\pi}}{(n / 2) !} \sqrt{\frac{n !}{2^{n-1}\left(2 \alpha^{2}+1\right)}}\left(\frac{2 \alpha^{2}-1}{2 \alpha^{2}+1}\right)^{n / 2}, \\
& B_{n}=B_{n}(\tau)=\frac{\sqrt[4]{\pi}}{\sqrt{2^{n-1} n !\left(2 \alpha^{2}+1\right)}}\left(\frac{2 \alpha^{2}-1}{2 \alpha^{2}+1}\right)^{n / 2} \times \\
& \times \exp \left(-\frac{\alpha^{2} \tau^{2}}{2 \alpha^{2}+1}\right) H_{n}\left(\frac{2 \alpha^{2} \tau}{\sqrt{4 \alpha^{4}-1}}\right) .
\end{aligned}
$$

In Fig. 1 we show the dependences of the mean error probability of distinction $P_{e}$ of signals $s_{1}(x)$ and $s_{2}(x)$ calculated with using Eq. (18), (19) for $h_{0}=0, p_{1}=p_{2}=0,5, \alpha=1, \tau=2$. Curve 1 corresponds to a processing of first two modes of signals $(M=1)$, curve 2 to a processing of first three modes $(M=2)$ and curve 3 to a processing of first five modes $(M=4)$. Here the limiting values of probability $P_{e}(15)$ at optimal reception $(M=\infty)$ by circles are also marked. From Fig. 1 it is obvious that the account of the several first modes of expansion of useful signals $s_{1}(x)$ and $s_{2}(x)$ provides the characteristics of distinction close to limiting.

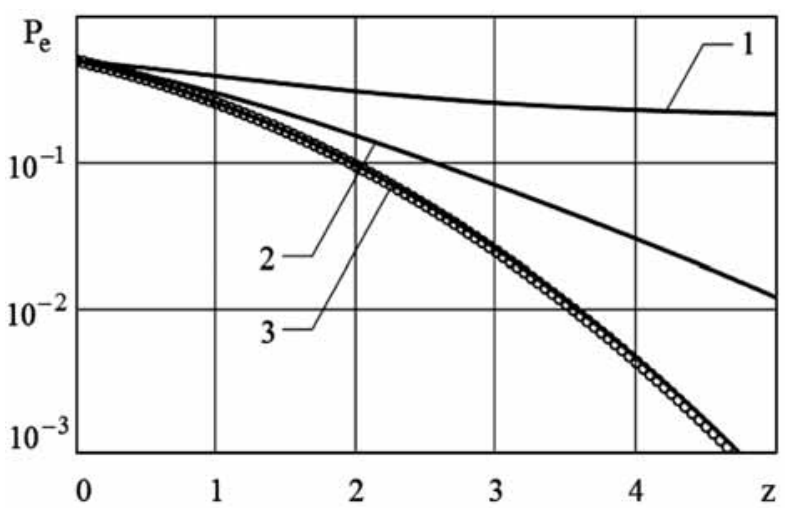

Fig. 1 Dependences of the mean error probability of distinction $P_{e}$ of signals $s_{1}(x)$ and $s_{2}(x)$

Let's put now that the reference signal of the optimal receiver contains also the limited number of modes, i.e. the structure of distinction algorithm is defined by a rule (8) and $\mathrm{M}>v$. In this case the logarithm of FLR (8) is a Gaussian random value with mathematical expectation and variance

$$
\begin{aligned}
& m_{1 L}=\left(E_{1 v}+E_{2 v}-2 R_{v} \sqrt{E_{1 v} E_{2 v}}\right) / N_{0}, \\
& \sigma_{1 L}^{2}=2\left(E_{1 v}+E_{2 v}-2 R_{v} \sqrt{E_{1 v} E_{2 v}}\right) / N_{0}
\end{aligned}
$$

at the hypothesis $H_{1}$ or

$$
\begin{aligned}
& m_{2 L}=-\left(E_{1 v}+E_{2 v}-2 R_{v} \sqrt{E_{1 v} E_{2 v}}\right) / N_{0}, \\
& \sigma_{2 L}^{2}=2\left(E_{1 v}+E_{2 v}-2 R_{v} \sqrt{E_{1 v} E_{2 v}}\right) / N_{0}
\end{aligned}
$$

at the hypothesis $H_{2}$. Here $R_{v}=\int_{\Omega} s_{1 v}(r) s_{2 v}(r) d r / \sqrt{E_{1 v} E_{2 v}}$.

From Eqs. (23), (24) it is easy to see that

$$
m_{1 L}=-m_{2 L}=m_{v}, \sigma_{1 L}^{2}=\sigma_{2 L}^{2}=\sigma_{v}^{2}, \sigma_{v}^{2}=2 m_{v} .
$$

Then for the mean error probability of distinction $P_{e}$ we can write down similarly Eq. (19):

$$
\begin{aligned}
& P_{e}=p_{1}\left[1-\Phi\left(\frac{h_{0}}{\sqrt{2 m_{\nu}}}\right)+\sqrt{\frac{m_{\nu}}{2}}\right]+ \\
& +p_{2} \Phi\left(\frac{h_{0}}{\sqrt{2 m_{\nu}}}-\sqrt{\frac{m_{\nu}}{2}}\right)
\end{aligned}
$$

The formula (25) becomes simplest at $p_{1}=p_{2}=0,5$ and $h_{0}=0$ :

$$
\begin{aligned}
& P_{e}=1-\Phi\left(\sqrt{\frac{E_{1 \nu}+E_{2 \nu}-2 R_{\nu} \sqrt{E_{1 \nu} E_{2 \nu}}}{2 N_{0}}}\right)= \\
& =1-\Phi\left(\frac{\sqrt{z_{1}^{2} \varepsilon_{1 \nu}+z_{2}^{2} \varepsilon_{2 \nu}-2 z_{1} z_{2} R_{\nu} \sqrt{\varepsilon_{1 \nu} \varepsilon_{2 \nu}}}}{2}\right) .
\end{aligned}
$$

If, besides, $\varepsilon_{1 v}=\varepsilon_{2 v}=\varepsilon_{v}, z_{1}=z_{2}=z$ then

$P_{e}=1-\Phi\left[z \sqrt{\varepsilon_{v}\left(1-R_{v}\right)} / 2\right]$.

In Eqs. (26), (27) it is designated $\varepsilon_{i v}=E_{i v} / E_{i}, i=1,2$, and variable $z_{i}$ is defined the same as in Eq. (14). From comparison of Eqs. (15) and (27) it is possible to conclude that incomplete reception (because of restrictions in mode structure of the receiver's reference signal. i.e., as consequence, condition performance $\varepsilon_{v}<1$ ) bring loses of optimal reception generally.

In Fig. 2 the dependences $P_{e}=P_{e}(z)$ calculated with use of formula (26) for signal models $s_{1}(x)(22)$ and $s_{2}(x)=s_{1}(x-\tau)$

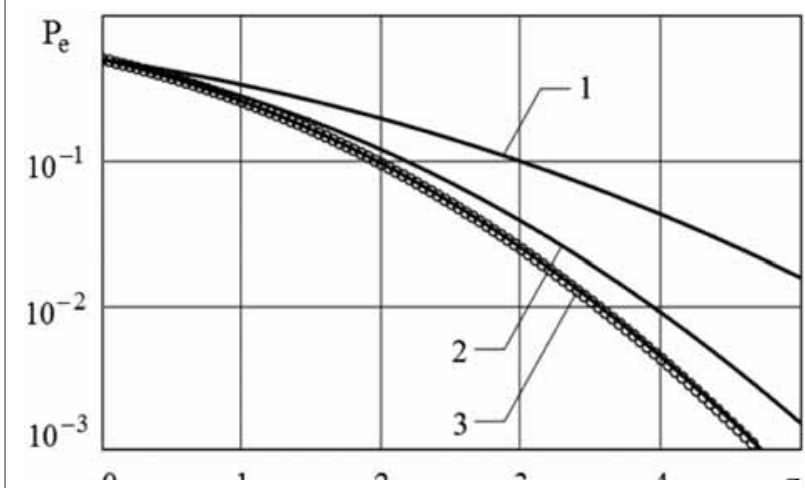

Fig. 2 Dependences $P_{e}=P_{e}(z)$ calculated with use of formula (26) 
at $\alpha=1, \tau=1$ and various numbers of processed modes $v$ are shown. Curve 1 is plotted at $v=1$ (the reference signal contains two modes), curve 2 at $v=2$ (the reference signal contains three modes) and curve 3 at $v=4$ (i.e., five modes of the reference signal are taken into account).

In Fig. 3 the similar dependences of probability $P_{e}(19)$ and (26) are represented for following variants of incomplete reception.

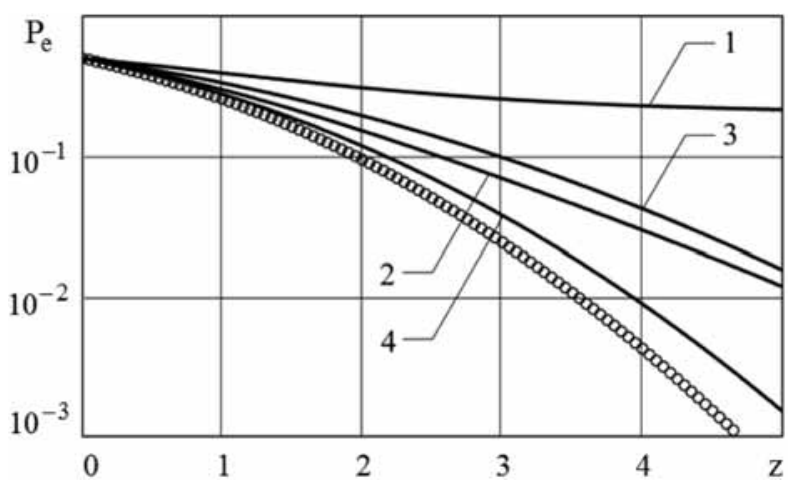

Fig. 3 Similar dependences of probability $P_{e}(19)$ and (26)

Curves 1 and 2 show the mean error probability of distinction when the sensed signal has two or three modes. Curves 3 and 4 are plotted for a case of restriction of the reference signal consisting of two (curve 3) or three (curve 4) modes. Circles in Figs. 2, 3 map limiting values of the probability $P_{e}(15)$ at optimal reception.

From graphs shown in Fig. 3 it is obvious that restrictions on number of modes in the reference signal are more advantageous energetically than restrictions in the sensed signal. This is due to the fact that if restrictions of the sensed signal occur then a loss of a part of useful signal energy takes place while noise energy remains invariable. And vise versa if restrictions of the reference signal occur that not only useful signal energy but also noise energy decreases on the receiver output.

\section{Conclusion}

At synthesis of distinction algorithms of signals and images their representation in the form of a set of the generalized spectrum's factors can appear rather effective. The given representation allows to receive simpler and practically realized discriminators of signals and images observed on the noise background. For strongly correlated signals and images the use of final mode number of sensed signals/images can provide smaller sacrifices of distinction quality in comparison with a case of distinction of full signals/ images. An incomplete reception of a useful signal causes loses of optimal reception in general because of restrictions in mode structure of a reference signal. However, the mode restrictions in a reference signal are more advantageous energetically (at the expense of reduction not only energy of a useful signal but also noise energy on a receiver output) than corresponding restrictions in the sensed signal. In addition, by expanding of useful signal to cover several first modes, this process can provide the distinction characteristics close to limiting.

The offered processing algorithms of signals and images can be almost realized with use of modern element base on the basis of digital signal processors or programmed logic integrated chips.

\section{References}

[1] RADCHENKO, Y.: Research of Signal Recovery, Suppression and Processing Algorithms Based on Polynomial Transformations, Proc. of the $6^{\text {th }}$ World Multiconference of Systemic, Cybernetics and Informatics, Florida : Orlando, 2002., Vol. VIX: Image, Acoustic, Speech and Signal III., pp. 262-266.

[2] RADCHENKO, Y.: Image Compression Algorithm on a Basis of Polynomial Transformations [in Russian], Digital signal processing, No. 1, 2002, pp. 2-6.

[3] BOLKUNOV, A. A., RADCHENKO, Y., OVCHINNIKOV, E. V.: Research of Polynomial Spectral Algorithms of the Shift Vector Estimation of Images' Dynamic Fragments [in Russian], Radio Engineering, No. 5, 2007, pp. 86-89.

[4] Adaptation in Information Optical Systems, Edited by N.D. Ustinov [in Russian], Radio i Svyaz', Moscow, 1984.

[5] The Theory of Coherent Images, Edited by N. D. Ustinov [in Russian], Radio i Svyaz', Moscow, 1987.

[6] Systems of Machine Vision, Edited by A.N. Pisarevsky and A.F. Chernyahovsky [in Russian], Mashinostroinie, Leningrad, 1988.

[7] TRIFONOV A. P., SHINAKOV Y. S.: Joint Discrimination of Signals and Estimation of their Parameters against Background [in Russian], Radio i Svyaz', Moscow, 1986.

[8] FIHTENGOL’TZ, G. M.: Bases of the Mathematical Analysis: the Textbook for High Schools [in Russian], Lan', St.-Petersburg, 1999.

[9] Handbook of Mathematical Functions with Formulas, Graphs and Mathematical Tables, Edited by M. Abramowitz and I. A. Stegun, National Bureau of Standards, Applied Mathematics Series 55, USA, 1964.

[10] GULDAN, V., MARCOKOVA, M.: Orthogonal Polynomials and Related Special Functions Applied in Geosciences and Engineering Computations, Communications - Scientific Letters of the University of Zilina, vol. 12, No. 1, 2010, pp. 12-15. 